\title{
Peer Reviewers for the Journal of the American Board of Family Medicine in 2019
}

\author{
Marjorie A. Bowman, MD, MPA, Anne Victoria Neale, PhD, MPH, \\ Dean A. Seebusen, MD, MPH, and Phil Lupo, MLIS
}

\section{(J Am Board Fam Med 2020;33:164-167.)}

To our peer reviewers: We thank you for your time, expertise, and dedication to the Fournal of the American Board of Family Medicine ( $A A B F M)$.

$7 A B F M$ peer reviewers serve a vital role in contributing to the field of family medicine and to its scientific community. We take this opportunity to acknowledge and thank our peer reviewers for their support during the past year. In 2019, 449 individuals provided 544 peer reviews and advice regarding the suitability of articles for publication in the FABFM. Reviewers took an average of 19 days to submit a review.

\section{Top Reviewers}

Each of the manuscripts submitted to the FABFM is first reviewed by the editors and, if

Conflict of interest: The authors are editors of the $7 A B F M$. deemed suitable for peer review, is evaluated and scored by volunteer reviewers. In the list below, peer reviewers with an asterisk next to their name are our top reviewers for 2019 based on evaluation scores and the quantity of critiques provided.

\section{Seeking New Reviewers}

We also enjoy welcoming new peer reviewers into the fold. Peer review is a vital service that helps authors and researchers improve their work. Reviewers are always free to decline a request or to indicate periods of unavailability. If you would like to volunteer to review for the $7 A B F M$, please download and complete the Peer Reviewer Form, which can be accessed online at https://www.jabfm.org/content/information-reviewers. Then, e-mail it, along with your curriculum vitae, to jabfm@med.wayne.edu, or fax it to 313-5776718.

$\begin{array}{ll}\text { 2019 Peer Reviewers } & \\ \text { Ndidi Ogechi Ivy Abara } & \text { Angele Arthur } \\ \text { Adrienne Z. Ables } & \text { Thomas Stewart Atkinson } \\ \text { Dmitry Abramov } & \text { John S. Baer } \\ \text { Sonal Admane } & \text { Steffani R. Bailey } \\ \text { Sumit Agarwal } & \text { Matthew G. Barnes } \\ \text { Krishna A. Agarwal } & \text { Donald Barr } \\ \text { David C. Agerter } & \text { Roger Bean } \\ \text { Morhaf Al Achkar } & \text { Keisa Bennett } \\ \text { Steven Albert } & \text { George R. Bergus } \\ \text { Nishita Shah Amin } & \text { Maresi Berry-Stoelzle } \\ \text { Holly Andrilla } & \text { Juliann Binienda* } \\ \text { Ann Gene G. Anthony* } & \text { Rajshekhar Bipeta } \\ \text { Kimberly Aparicio } & \text { Alan Blum* } \\ \text { Christine Arenson } & \text { Debra Boardley } \\ \text { Brian Arndt } & \text { George W. Bock* }\end{array}$

John M. Boltri

Curtis Bone

Jason C. Booza*

Jeffrey M. Borkan

Amanda E. Borsky

Deborah Bowen

Marie Teresa Brown

Christopher W. Bunt

Lucinda M. Buys

Walter L. Calmbach

Gregory Camilli

Kimberly D. Campbell-Voytal

Doug Campos-Outcalt

Peter J. Carek

Timothy S. Carey 


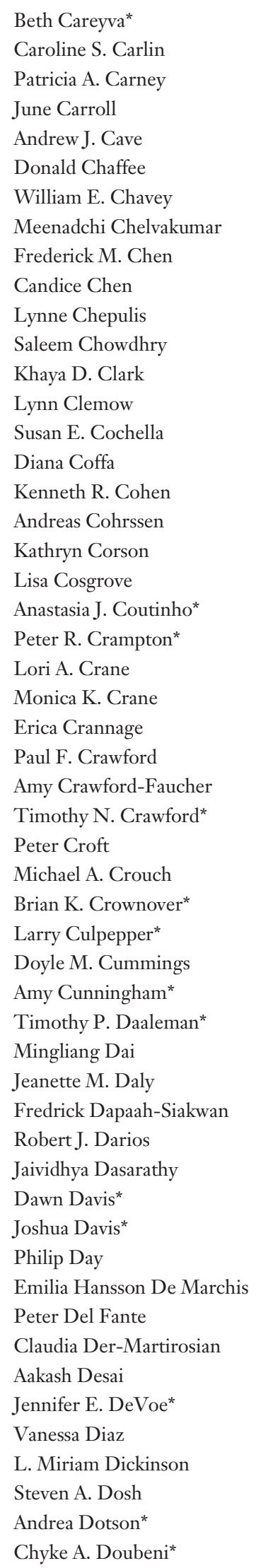

Deborah Dreyfus

Dora M. Dumont

Samuel Edwards

Ellen M. Einterz

Nancy C. Elder*

John W. Epling**

Philip M. Eskew

Anna Espinoza*

Rebecca S. Etz

Heather B. Fagan

Krista Farey

Bryan Farford

Anum Fasih

Steven R. Feldman

Jeanne M. Ferrante

Robert L. Ferrer

Scott A. Fields

Edgar Figueroa*

Alexander G. Fiks

Kenneth S. Fink*

Kevin Fiscella

Rebecca Fischbein*

Judith A. Fisher

Margaret Flinter

Jonathan Foley*

Kanti R. Ford

John Fox

Gary Franklin

Adam Franks*

Dan Frayne

Joshua Freeman

John J. Frey

Kimberly G. Fulda*

Heidi L. Gaddey

Anne H. Gaglioti

Sim S. Galazka

Jinal Gangar

David S. Garcia*

Gregory M. Garrison

Sarah Gebauer

Lekha K. George

Cynthia M. Geppert

Jenenne Geske

Valerie J. Gilchrist

Sarah L. Goff

Marji Gold

Stephanie B. Gold

Debora Goetz Goldberg

Rise B. Goldstein

Thomas Granatir

Lee A. Green

Larry A. Green*

Beverly Green*
Grant M. Greenberg*

Kevin Grumbach

Alexander B. Guirguis

Karen Gunning*

Tamara M. Haegerich

Kristin Haglund

David L. Hahn

Jacqueline R. Halladay

Robert M. Hamm

E. Haneke

Susan Hart-Hester

Robert L. Hatch

Clare Hawkins

Jennifer Hefner

Diana L. Heiman

Robin Helm

Margaret Helton

William G. Henderson

Paul J. Hershberger

Vincent $W$. Hevern

John Hey

Paul C. Hicks

Andrew Holdaway

Neil R. Holland

Jakob Holstiege

James D. Holt

Jodi S. Holtrop

Michael A. Horst

Susan Hsieh

Clarissa Hsu

Hsiang Huang

Nathalie Huguet

Kenya Ie

Jihad Irani*

Oluwaseyi O. Isehunwa

Jamal Islam

Henry R. Ivey*

Akila Iyer

Yalda Jabbarpour

Raja Jaber

Masahito Jimbo*

Jeremy D. Johnson

Andrew T. Jones

Bonnie T. Jortberg

Neelima Kale

Medha Karmarkar

David Katerndahl

Lisa C. Kellar

Sara Keller

Rodger Kessler*

Omar A. Khan

Niharika Khanna

Autumn M. Kieber-Emmons 


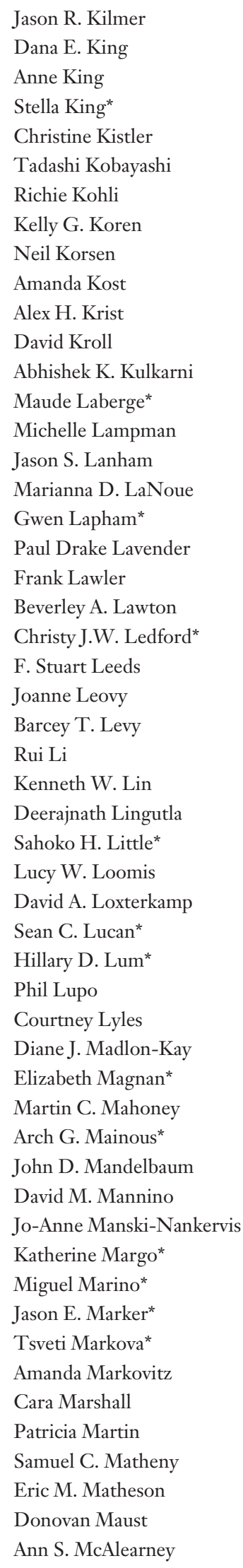

Kerry McBrien

Catherine McCarthy

Paul Meissner

John D. Mellinger

Rowena Merritt

Ashley Meyer

David Meyers

F. Matthew Mihelic

William Miller

Virginia Miller

Logan Mims

Tasaduq Mir

Joi L. Moore

Miranda Moore*

Laura Morgan-Frankart

Christopher P. Morley*

Karoline Mortensen

Cortney M. Mospan*

Karen Muchowski*

Maureen A. Murtaugh

Elanagan Nagarajan

Zsolt J. Nagykaldi*

Priya Nambisan

Jatinder Narang

Raj Narayan

Susan Nash

Terence Dwight Naumann

Donald E. Nease*

Andrea Lynn Nederveld

Lynne S. Nemeth

Giang T. Nguyen

Kari Nilsen

Thomas E. Norris

Thomas F. Northrup

Mary Patricia Nowalk

David Nowels

Maeve O'Beirne

Alane B. O'Connor

Jason O'Grady

David T. O'Gurek

Thomas R. O'Neill

Cormac John O'Connor

Robert C. Oh

N. I. Okpokwasili

Eriko Onishi

Amimi Sandra Osayande

Darrell R. Over

Lynda J. Owen

Wilson D. Pace

Bethany Panchal

Michael L. Parchman

John M. Pascoe

Donald Pathman*
Nikita Patil

Ganesh Pawar

Julie Petersen

Lars Peterson

Stephen Petrany*

James Phelps

William R. Phillips

Julie Phillips

Lusine Poghosyan

Charles D. Ponte*

Michael B. Potter

David W. Price

James C. Puffer

Perry A. Pugno

Marsha A. Raebel

Barbara M. Rainer

Meghan F. Raleigh

Ravishankar Ramaswamy

Anita Ravi

Brian V. Reamy*

Joanne L. Reeve

Jo Marie Reilly

Peter L. Reynolds*

Robert L. Rhyne

Jill Rissi

Peter A. Rives

Pasquale Roberge

Pamela G. Rockwell

Michelle A. Roett

Leonardo Roever

Max Romano

Adam J. Rose

Jack M. Rosenberg

Stephanie E. Rosener

Thomas C. Rosenthal

Robert G. Ross

Mack T. Ruffin

Roy T. Sabo

Joseph Sakai

Enrique Sanchez Valdivieso

Paulo A. Santos

Amit Sapra

Amelia Sattler

John W. Saultz

Judith A. Savageau*

Margot Savoy

Stanley Schaffer

Kenneth G. Schellhase

Joseph E. Scherger

Nicholas B. Schmuhl

Eugene P. Schoener

Sarina Schrager

Kendra Schwartz 


Miriam E. Schwartz
H. R. Searight
Elizabeth Seiverling
Kevin Selby
Jessica Servey*
Patricia S. Sexton
Patricia L. Seymour
Anjana E. Sharma
Jonathan Shaw
Eric K. Shaw
William J. Sieber
Matt Silvis
Gina Simoncini
Hardeep Singh
Gurmukh Singh
Eman Sobh
Leif I. Solberg
Yazhini Srivathsal
Joshua St. Louis
Elizabeth W. Staton
Kenda Stewart Steffensmeier
Keith Stelter
Nancy G. Stevens
Earl Ray Stewart
Michael A. Stoto
Mark L. Stovak
George D. Strelioff
Courtney Kimi Suh
Mark D. Sullivan

Walton Sumner

Craig Surman

John E. Sutherland

Daniel J. Tancredi*

Hazel Tapp*

Derjung M. Tarn

Yhenneko J. Taylor

Sharon Ternullo

Chris M. Terpening*

Manish M. Tiwari

Barbara Tobias

Rachelle Toman*

Sebastian T. Tong

Stefan Topolski

Dennis Tsilimingras

Ericka E. Tung

Marc Tunzi

Mark J. Upfal

Carole C. Upshur

Anthony F. Valdini

Carla Veiga Rodrigues

William B. Ventres

Mohamud A. Verjee

Bonnie M. Vest

Karen M. Vitale

Nicki Vithalani

Kirsten Vitrikas

Daniel Waldman

Eric Wall*
Anne D. Walling

Richard C. Wasserman

Donald Weaver

Sally Weaver

Toney Welborn

David L. Weldy

Mary Jo Welker

Karen Wernli

Karen M. Whalen

John Sharkey White

Brett White

Elizabeth Wickersham

Rachel Willard-Grace

Summer Williams

Robert L. Williams

Thad E. Wilson

Scott Wiltz

Diana Wohler

Jennifer Wolff

Michelle Wong

Anne B. Woods

Melissa A. Wright

Imam M. Xierali*

Jinping $\mathrm{Xu}$

Bektas Murat Yalcin

Joseph R. Yancey

Jonathan Yun

Emily B. Zimmerman

*Top reviewers in 2019 (based on quantity and quality of peer reviews written).

To see this article online, please go to: bttp://jabfm.org/content/33/2/164.full. 